\title{
ELECTROCHEMICAL RESPONSE OF AA7075-T651 FOLLOWING IMMERSION IN NaCI SOLUTION
}

\author{
N.Birbilis, M.K. Cavanaugh, R.G. Buchheit \\ Fontana Corrosion Center, \\ The Ohio State University. \\ Columbus, $\mathrm{OH} 43210$.
}

\begin{abstract}
The electrochemical behavior of AA7075-T651 following immersion in quiescent $0.1 \mathrm{M} \mathrm{NaCl}$ is presented. Electrochemical impedance at various polarization intervals was determined using Fourier transformation of

potentiostatically induced current transients. This allowed for rapid determination of the impedance response at fixed intervals revealing a more detailed insight into the kinetic response of the alloy when assessed with complementary analysis tools such as potentiodynamic testing. This led to a discussion regarding aspects of dissolution phenomena prior to alloy breakdown and at short immersion times.
\end{abstract}

\section{INTRODUCTION}

The last two decades have seen extensive investigations into the corrosion behavior of high strength aluminum alloys (1-8), however certain aspects regarding electrochemical characterization of such alloys have not yet been resolved; i.e. the impedance characteristics across a range of potentials.

It is widely accepted that AA7075 has poor corrosion resistance owing largely to a significant population of second phase intermetallic particles, which may be anodic or cathodic relative to the alloy matrix and are synonymous with particle induced pitting (58). Common intermetallics in AA7075 include $\mathrm{MgZn}_{2}, \mathrm{Mg}_{2} \mathrm{Si}, \mathrm{Al}_{3} \mathrm{Fe}, \mathrm{Al}_{7} \mathrm{Cu}_{2} \mathrm{Fe}$, $\mathrm{Mg}(\mathrm{AlCu})$ and $\mathrm{Al}_{2} \mathrm{CuMg}$, the individual electrochemical characteristics of which have been presented in $(7,9)$. This recent work regarding the electrochemical characteristics of the constituents of AA7075 on a phase-by-phase basis along with other fundamental work characterizing AA7075-T651 microstructure now allows for greater interpretation of the response of the bulk alloy. Furthermore, recent advances in methodologies aimed at monitoring and interpreting complex electrochemical systems have also advanced (1013).

Upon immersion in neutral $\mathrm{NaCl}$ solution (quiescent), the difference between the breakdown potential and corrosion potential $\left(\mathrm{E}_{\mathrm{pit}}-\mathrm{E}_{\mathrm{corr}}\right)$ as observed by potentiodynamic testing is generally below $100 \mathrm{mV}$ for AA7075 (6); however, with sufficiently longer exposure times $\mathrm{E}_{\mathrm{pit}}=\mathrm{E}_{\text {corr }}$ and corrosion occurs freely (2). In this work we will investigate the electrochemical response, namely impedance characteristics, of AA7075T651 during the early stages of immersion in $\mathrm{NaCl}$. The motivation for determination of electrode impedance in the case of Al alloys is driven by the need to develop a more comprehensive insight into the corrosion process than that given by $\mathrm{E}_{\text {corr }}$ or $\mathrm{E}_{\text {pit. }}$ For example, the relative nobility of $\mathrm{E}_{\mathrm{pit}}$ does not necessarily correlate with the extent of 
pitting observed $(2,10)$. Furthermore, following corrosion initiation, an accurate assessment of kinetic processes may be of significant importance in the understanding of the mode and morphology of corrosion damage accumulation, the corresponding extent of damage accumulation, and the efficacy of any remedial measures (such as the application of inhibitors).

The electrochemical impedance of alloys such as AA7075 in $\mathrm{NaCl}$ solutions has proven difficult to monitor by the classical impedance method, owing to their nonstationary response. Systems that are prone to pitting in the solution in which they are immersed reveal time dependant behavior (due to their altering character as pitting proceeds); whilst also being sensitive to perturbation (viz. the properties of the electrode change as a result of the applied polarization). As a result, the electrochemical impedance collected upon such systems will not necessarily represent the true electrode response, whilst being ambiguous in the investigation of pitting process, leading to qualitative and not quantitative results being obtained.

An example of this may be seen in Fig. 1, whereby a Nyquist plot for AA7075T651 is seen following $1 \mathrm{~min}$ and $60 \mathrm{mins}$ immersion in $0.1 \mathrm{M} \mathrm{NaCl}$. The inductive loop observed at low frequencies is indicative of a non-linear response (not necessarily representing the ratio of sinusoidal signals), ultimately yielding a system response and not a true impedance. Fig. 2 shows the Bode plot corresponding to Fig. 1 where it becomes evident that at low frequencies the impedance magnitude appears to be decreasing with a concomitant positive phase angle; this suggests that corrosion activity is likely intensifying during the measurement necessitating an significant increase in the current required to generate a given potential shift.

The non-stationary character of AA7075 was previously noted by Mansfeld (1), who postulated that the anodic portion of the AC signal caused initiation or acceleration of localized corrosion and in order to determine the impedance response of 7075 , suggested recording EIS data (following immersion in $\mathrm{NaCl}$ ) at $\mathrm{E}_{\text {corr }}-20 \mathrm{mV}$. However, such an approach cannot be considered a general course of action in order to avoid errors associated with non-stationary electrode behavior. In general, the time taken to complete a conventional EIS measurement and the magnitude of the cumulative charge passed during the measurement are the factors responsible for perturbing the system. This concept has been emphasized in some detail recently, and there has been an effort to develop methodologies that may permit for accurate determination of impedance upon non-stationary systems (10-16), such as Fourier Transform Electrochemical Impedance Spectroscopy (FT-EIS) (16) or dynamic EIS (10-11). Such methods are attractive as they can allow for large reductions in the time taken to complete a measurement (minimizing the amount of electrode perturbation) and may also allow for impedance information to be collected over a range of potentials separated from $E_{\text {corr }}$, or even under open circuit conditions, without demanding a stationary electrode response. The basis for such methods is largely founded on the fundamental principles outlined by Macdonald and McKubre (15), which highlighted the relationships that exist between voltage and current required to define interfacial impedance, noting that such relationships are not confined to the frequency domain alone and that transient methods yield the same information in principle. 
An approach for determining electrochemical impedance of AA7075-T651 dynamically from data collected in the time domain

It was decided to use a (transient) time domain approach to determine the electrode impedance $(Z(j \omega))$ for a finite analysis window, whilst facilitating the ability to generate data over a range of potentials. This involved determination of the frequency domain response via Fourier transformation (FT) of the time domain response, according to (15). The frequency domain response via Fourier transformation (FT) of the time domain response, can be given by the general expression;

$$
Z(j \omega)=\frac{E(j \omega)}{I(j \omega)}=\frac{F T[E(t)]}{F T[I(t)]}
$$

The FT of a continuous time function $I(t)$ on a finite time interval $[0, T]$ is defined by:

$$
F T[I(t)]=I(f) \equiv \int_{0}^{T} I(t) e^{-j 2 \pi \cdot f \cdot t} d t
$$

Where $j=\sqrt{-1}, f$ is the frequency in $\mathrm{Hz}$, and $2 \pi \cdot f=\omega$.

Eqn. 2 can be solved by a number of simple numerical methods. When $I(t)$ is sampled at discrete evenly spaced time intervals, $I(f)$ may be approximated by:

$$
F T[I(t)]=I(f) \approx \Delta t \sum_{i}^{N-1} I_{i} e^{-j 2 \pi \cdot f \cdot t_{i}}
$$

Where $t_{i}=i \Delta t, I_{i}=I(i \Delta t), i=0,1,2, \ldots \mathrm{N}$.

The nature of this transformation allows for the output of a complex response containing 'real' and 'imaginary' data, which must fall within the frequency band given by $\left[0, f_{n}\right]$, where;

$$
f_{n}=\frac{1}{2 \Delta t}
$$

This transformation approach considers the response as discrete data points rather than a continuous function. The rate at which the response is sampled and the duration over which it is sampled determine the extent of frequency domain that is resolvable. By selecting a given E-I window to register, one can generate a set of impedance information reflecting the dynamics of the process examined.

\section{EXPERIMENTAL}

Testing was performed on AA7075-T651 (supplied by Alcoa). The T651 temper designation refers to a solution treated and artificially aged (to peak hardness) alloy, followed by a stress relief ( $0.5-3 \%$ stretching) treatment. The surface of the alloy was polished to a $1 \mu \mathrm{m}$ finish using successive steps, finishing with a diamond paste / alcohol suspension.

An Autolab potentiostat (PGSTAT 100) under the control of GPES V4.9.4 software was used. In other cases, a Gamry PC-3 potentiostat was used. In all cases a three-electrode 'flat-cell' arrangement was used exposing $1 \mathrm{~cm}^{2}$ of electrode. Quiescent $0.1 \mathrm{M} \mathrm{NaCl}(\mathrm{pH} \mathrm{6})$ solution was used for electrochemical testing. When a potentiostatic staircase signal was used, sample immersion time was 5 minutes prior to testing, each potential was sustained for a period of $10 \mathrm{~s}$, and along with the associated current, the recorded signal were subsequently Fourier transformed (in this case using an FFT algorithm (17)) to generate the electrode impedance. The frequency range determined by this approach yields results between $50 \mathrm{~Hz}$ and $0.2 \mathrm{~Hz}$. A cautionary note should be made 
here to emphasize that the current transient resulting from a potentiostatic signal is influenced rather significantly by the electrolyte resistance (ohmic resistance, $\mathrm{R}_{\Omega}$ ) (18), thus the aforementioned approach is suited toward systems with negligible electrolyte resistance such as the case investigated here. Where potentiodynamic polarization was used, a potential scan rate of $1 \mathrm{mV} / \mathrm{s}$ was employed. Conventional impedance spectra were collected at $\mathrm{E}_{\text {corr }}+/-10 \mathrm{mV}$ over a range of $5 \mathrm{kHz}$ to $20 \mathrm{mHz}$. In cases where fitting of impedance data was done, the program ZSimpWin was used. Microscopy was carried out using a Philips TF-20 FEG operating at $200 \mathrm{kV}$.

\section{RESULTS and DISCUSSION}

\section{Electrochemical response of AA7075-T651}

The typical current response to an anodic potentio-staircase signal is seen in Fig. 3. We observe that each incremental potential step results in a current transient that may be subsequently used to define the electrode impedance. As the amount of polarization increases, we see an ensuing rise in the measured current.

Prior to presenting the evaluated impedance response of the alloy at each potential increment, we may view the potential plotted along with the corresponding current response (taken following 10s from the application of the potential step). Fig. 4 shows this response (restricted to the potential window of $E_{\text {corr }}+/-0.35 \mathrm{~V}$ ) along with the corresponding potentiodynamic polarization curve for AA7075-T651. The data collected using the potentio-staircase signal correlate well with those determined by the potentiodynamic scan. It should be noted that the potentio-staircase data represents two tests; one yielding the cathodic data (starting from $\mathrm{E}_{\text {corr }}$ and increasing the polarization in the cathodic direction which is not discussed in this paper), the other the anodic polarization data respectively. There is some disparity between the two techniques at large cathodic overpotentials; however, this is attributed to overestimation of current in the case of the potentiodynamic test, since testing in that case was conducted (swept in the anodic direction) from $-1.1 \mathrm{~V}_{\mathrm{SCE}}$ and the initial data points may be influenced by charging of the double layer capacitance.

Fig. 4 highlights such an approach for subsequently determining the impedance of the alloy at incremental potential steps may be considered somewhat equivalent to probing the impedance response at discrete locations upon the polarization curve of the alloy. Consequently, in the following section, we present and discuss the impedance evaluated from the 10 s current transient at these discrete potential intervals.

$\underline{\text { Investigation of electrode impedance during anodic polarization }}$

The Nyquist representation of the impedance response of AA7075-T651 determined from Fourier analysis following the application of a potentiodynamic staircase signal is seen in Fig. 5. $\Delta \mathrm{E}_{\text {anod }}$ represents the amount of anodic polarization from $E_{\text {corr }}$ that the electrode has been subjected to for the 10 s window used to determine $(Z(j w))$.

From Fig. 5, we observe the largest value of impedance measured occurred at the lowest anodic overpotential, $0.025 \mathrm{~V}$, and decreased several orders of magnitude 
following $\Delta \mathrm{E}_{\text {anod }}>0.1 \mathrm{~V}$, concomitant with a rapid rise in current (Fig.3).

The approach adopted in this study allows for investigation of the impedance response prior to large-scale alloy breakdown (viz. $\mathrm{E}<\mathrm{E}_{\mathrm{pit}}$ ), revealing kinetic characteristics for the evolution of corrosion under potentiostatic conditions following immersion in $0.1 \mathrm{M} \mathrm{NaCl}$.

In order to model the response of a metal susceptible to pitting, a number of researchers (2-4) have adopted use of the model of the form given in Fig. 6. The electrode area fraction associated with the first branch of the circuit (incorporating $R_{1}$ ) is given as $(1-F)$, thus the electrode area associated with the second branch (incorporating $R_{2}$ ) is given as $\mathrm{F}$. In such cases $\mathrm{F}$ represents the area fraction of pitted surface. Q is a 'constant phase element' (CPE), included to facilitate the possibility of a capacitance that is not precisely Debye-like (20) via the exponent $\beta$, where $\beta=1$ represents ideal capacitance, $\beta$ $=0.5$ yields a linear response with decreasing frequency and $0.5<\beta<1$ represents a stretched exponential. W is the Warburg impedance (20).

As a consequence of the above notion, in the absence of pitting, a simple 'Randle's type' circuit with the possibility of incorporating a CPE (viz. $\mathrm{R}_{\Omega}\left(\mathrm{R}_{1} \mathrm{Q}_{1}\right)$ ) should be sufficient to characterize the electrochemical response of the metal investigated when $\mathrm{F}=0 . \mathrm{F}$ is nominally taken as 0 prior to stable pitting, and thus, $\mathrm{F}>0$ should be reserved for situations where breakdown has been realized (either by long term immersion or by polarizing beyond $\mathrm{E}_{\mathrm{pit}}$ ). From Fig. 5 (and later Fig. 8), however, it is apparent that at low anodic overpotentials below $\mathrm{E}_{\mathrm{pit}}$, a Randle's type (single time constant) circuit is not capable of characterizing the system investigated, owing to the complicated form of the evaluated impedance response.

In this work, we have noted that it is more appropriate to use the circuit of Fig. 6 to model the electrochemical impedance of the system prior to breakdown. For the case of $\Delta \mathrm{E}_{\text {anod }}<0.1 \mathrm{~V}$, the circuit of Fig. 6 gave a satisfactory fit.

The presence of two distinct processes occurring over different frequency ranges prior to breakdown reveals behavior of AA7075-T651 is richer than simple 'passive' or 'active' characterization.

\section{Discussion of (anodic) dissolution of AA7075-T651}

The notion of so-called 'transient' dissolution prior to breakdown was recently presented but not discussed in detail (6). In essence, the electrochemical dissolution of active species/components in the alloy, prior to the observed $\mathrm{E}_{\text {pit }}$, can go some way in explaining the form of the impedance response seen in Fig. $5\left(\Delta \mathrm{E}_{\text {anod }}<0.1 \mathrm{~V}\right)$.

Other fundamental work aimed at electrochemical characterization of individual intermetallics in AA7075 on a phase by phase basis (7) has been carried out and reveals significant anodic dissolution of specific intermetallics present in AA7075 at potentials $<<\mathrm{E}_{\text {pit }}$ (of AA7075-T651). These intermetallics include $\mathrm{MgZn}_{2}, \mathrm{Mg}_{2} \mathrm{Si}, \mathrm{Al}_{32} \mathrm{Zn}_{49}, \mathrm{Mg}_{2} \mathrm{Al}_{3}$ and possible dealloying of $\mathrm{Al}_{2} \mathrm{CuMg}$ (7). Such dissolution prior to breakdown does not exclusively refer to metastable pitting events, which have been noted as occurring as a prelude to large-scale breakdown in other systems (26), but dissolution without the possibility of repassivation (of the intermetallic). This notion is emphasized in Fig. 7, whereby the potentiodynamic polarization curves for $\mathrm{MgZn}_{2}$ and $\mathrm{Mg}_{2} \mathrm{Si}$ show the ability to sustain large anodic current densities at potentials typical of AA7075-T651 in $\mathrm{NaCl}$ solution (i.e. in the vicinity of $-0.8 \mathrm{~V}_{\mathrm{SCE}}$, discussed further below).

Based on investigations of the alloy microstructure, the most abundant of the (anodic) intermetallics is $\mathrm{MgZn}_{2}$, followed by $\mathrm{Mg}_{2} \mathrm{Si}$ (6, 21-22). With regard to 
stereological considerations, such intermetallics are present in an abundance capable of influencing the electrochemical characteristics of the alloy (viz. approximately $100 / \mu^{2}$ (22)).

Since compounds such as $\mathrm{MgZn}_{2}$ and $\mathrm{Mg}_{2} \mathrm{Si}$ are not capable of forming a passive film in $\mathrm{NaCl}$ solutions, their dissolution ought to be essentially spontaneous and not manifest as a 'breakdown' phenomena. It is posited that the dissolution of these active/anodic intermetallics is responsible for the form of $Z(j w)$ observed prior to breakdown. The typical form of $Z(j w)$ prior to breakdown (Fig. 8a) reveals that it is difficult to discern multiple processes corresponding with the individual type of intermetallics that may be active. Instead, there are two distinct processes; one likely representing the 'active' components distributed over the alloy and the other representing the matrix/noble components.

Following breakdown, $\Delta \mathrm{E}_{\text {anod }}>0.1 \mathrm{~V}$, the form of $Z(j w)$ may be represented simply by the modified Randle's circuit (Fig. 8b) including a Warburg term (i.e. Fig. 6 without the $\mathrm{R}_{1} \mathrm{Q}_{1}$ branch). This again indicates that it is difficult to discern between the response of individual classes of features that are undergoing pitting (viz. various intermetallics or matrix) and that the impedance response is dominated by all corroding sites equally, which are also likely paths of least resistance and most efficient at drawing the current. This observation corresponds with the work of Conde (3) performed upon aluminum alloy 2024. Instead of multiple time constants, the CPE term accounts for some dispersion in the response of electrode (viz. $0.5<\beta<1$ ). It should be mentioned that the lack of multiple processes following breakdown may suggest that noble intermetallics (viz. $\mathrm{Al}_{2} \mathrm{Cu}, \mathrm{Al}_{7} \mathrm{Cu}_{2} \mathrm{Fe}, \mathrm{Al}_{3} \mathrm{Fe}$ and $\mathrm{Mn}$ containing particles (7)) no longer have a significant impact on the electrochemical response of the system under potentiostatic conditions, although they may well be associated with 'peripheral' pitting of the matrix under open circuit conditions (8).

Further evidence for the loss of anodic phases prior to large scale breakdown of the alloy can be ascertained by inspection of the open circuit potential (OCP) of AA7075T651 following immersion in $\mathrm{NaCl}$ solutions of varying concentration (Fig. 9). As time proceeds, the OCP evolves to more noble values. Based on the previous discussion, it is postulated that this may possibly be attributed to loss of $\mathrm{MgZn}_{2}$ and $\mathrm{Mg}_{2} \mathrm{Si}$. This is rationalized by the fact that $\mathrm{MgZn}_{2}$ and $\mathrm{Mg}_{2} \mathrm{Si}$ have their own open circuit potential values of about -1.03 and $-1.4 \mathrm{~V}$, respectively, in $0.1 \mathrm{M} \mathrm{NaCl}$ (7). Hence, the loss of such phases should lead to ennoblement of the alloy under open circuit conditions (similar to the manner seen in Fig. 9) towards values approaching -0.8 to $-0.75 \mathrm{~V}$.

Furthermore, it is interesting to note that when using the sample immersed in $0.1 \mathrm{M} \mathrm{NaCl}$ for $1000 \mathrm{~s}$ immersion (used to generate Fig. 9), subsequent potentiodynamic testing yields a distinct breakdown potential for the alloy (Fig. 10). This highlights that the loss of anodic phases prior to alloy breakdown may not likely have a marked impact on the $\mathrm{E}_{\mathrm{pit}}$ of the bulk alloy, especially if $\mathrm{E}_{\mathrm{pit}}$ is a manifestation of activation of the matrix phase exclusively.

In order to appreciate the microstructural impact of corrosion, it was decided to immerse an AA7075-T651 TEM specimen in $0.1 \mathrm{M} \mathrm{NaCl}$ for 1000 s. The result is seen in Fig. 11.

What we see is a discrete loss (dissolution) of $\eta$ type (i.e. $\mathrm{MgZn}_{2}$ ) particles. In this dark field image, regions where $\eta$ was lost appear as dark blemishes, due to localized thinning (in most cases the total absence) of the sample. Not all $\eta$ particles were dissolved, suggesting that those completely dissolved were either the smallest and / or those which intersected the surface (or underside) of the sample. We also see continuous 
dark zones along the grain boundary.

The main point to gauge from Fig. 11 is that $\eta$ particles appear to display the ability to behave as unique electrochemical entities. This reinforces the electrochemical results whereby the spontaneous dissolution of $\eta$ (and on larger length scales, $\mathrm{Mg}_{2} \mathrm{Si}$ ) ought to be expected (Fig. 7) and that this dissolution (on the sub $\mu \mathrm{m}$ level) does not impact bulk alloy breakdown (Fig. 10), suggesting that ultimate damage accumulation is developed by at least two distinct processes - one responsible for the dissolution of $\eta$ particles, the other responsible for matrix pitting. Following longer immersion times (3600s), we see that the polarization behavior of AA7075-T651 does not reveal a breakdown with high dissolution rates at $\mathrm{E}>\mathrm{E}_{\text {corr }}$.

It is prudent to state at this point that under open circuit conditions, large-scale attack of the matrix is often associated with 'peripheral' pitting around noble particles that serve as potent cathodes (5). Under potentiostatic conditions however, random pitting of the matrix may be seen $\left(\mathrm{E}>\mathrm{E}_{\mathrm{pit}}\right)$ owing to pit generation by electrochemical means at local 'weak-spots' in the passive film upon the matrix. These two abovementioned processes are independent of the transient dissolution mechanism of $\eta$ particles discussed in detail here, whilst occurring on nominally larger length scales.

In all, the combination of electrochemical techniques used herein has allowed us to reconcile dissolution phenomena on the bulk alloy following immersion in $\mathrm{NaCl}$ solution. Knowledge of the alloy microstructure together with details regarding active phases within the alloy has allowed us to tighten the microstructure-corrosion relationship from electrochemical signatures from bulk alloy testing, whilst pointing towards more future work.

Further to the above work, investigations into the cathodic kinetics and time dependant response of AA7075 were also carried out but will not be reported herein.

\section{CONCLUSIONS}

The approach adopted herein, whereby the application of a potentio-staircase polarization was used, is a convenient method for investigating the impedance response at unique potentials selected by the user. We showed that it was possible to determine the impedance behavior of AA7075-T651 across a range of potentials in a short timeframe via transformation of data collected in the time domain. The kinetic response of the system measured using this approach would not have been possible by classical EIS, since the processes probed, such as transient dissolution (in this case manifest as multiple time constants) prior to breakdown, occur too rapidly to be studied in any great detail by classical EIS. The electrochemical results were extended towards a discussion regarding alloy microstructure, and it was seen that $\eta$ particles display the ability to behave uniquely (and independent of the matrix) by dissolving at $\mathrm{E}<\mathrm{E}_{\mathrm{pit}}$. This phenomenon did not impact large-scale breakdown of the alloy, which was posited to be a consequence of breakdown of the matrix phase. Following long immersion times we see $\mathrm{E}_{\mathrm{corr}}=\mathrm{E}_{\mathrm{pit}}$. 


\section{REFERENCES}

1. F. Mansfeld, J.C.S. Fernandes, Corros. Sci. 34 (12), 2105 (1993).

2. F. Mansfeld, S. Lin, H. Shih, ASTM Special Technical Publication: STP-1134, ASTM, Philadelphia, PA., 141, (1992).

3. A. Conde, J. De Damborenea, Corros. Sci., 39 (2), 295 (1997).

4. F. Mansfeld, S. Lin, S. Kim, H. Shih, J. Electrochem. Soc., 137 (1) 78 (1990).

5. Z. Szklarska-Smialowska, Corros. Sci. 411743 (1999).

6. Q. Meng, G.S. Frankel, J. Electrochem. Soc., 151 (5), B271 (2004).

7. N. Birbilis, R.G. Buchheit, J. Electrochem. Soc., 152 (4), B140 (2005).

8. F. Andreatta, H. Terryn, J.H.W. de Wit, Electrochim. Acta, 49 (17-18), 2851 (2004).

9. R.G. Buchheit, J. Electrochem. Soc., 142, 3994 (1995).

10. K. Darowicki, S. Krakowiak, P. Slepski, Electrochim. Acta, 49 (17-18), 2909 (2004).

11. K. Darowicki, J. Orlikowski, A. Arutunow, Corrosion Engineering, Science and Technology, 39 (3), 255 (2004).

12. D.J. Gavaghan, D. Elton, K.B. Oldham, A.M. Bond, J. Electroanal. Chem., 512, 1 (2001).

13. J.E. Garland, C.M. Pettit, D. Roy, Electrochim. Acta, 49, 2623 (2004).

14. K. Sugimoto, Y. Masahiro, Bulletin of the Japan Institute of Metals, 23, 19 (1984).

15. D.D. Macdonald, M.C.H. McKubre, Impedance measurements in electrochemical systems, in: J. O’M. Bockris, B.E. Conway, R.E. White (Eds.), Modern aspects of electrochemistry No. 14, Plenum Press, New York, 1982, pp61-150.

16. S.J.M. Rosvall, M.J. Honeychurch, D.M. Elton, A.M. Bond, J. Electroanal. Chem., 515, 8 (2001).

17. R. Alvarez, M. Sanchez, Corrosion Reviews, 20, 69 (2002).

18. J.A. González, J.M. Miranda, N. Birbilis, S. Feliu, Corrosion, 61, 37 (2005).

19. G.S. Frankel, L. Stockert, F. Hunkeler, H. Boehni, Corrosion, 43 (7), 429 (1987).

20. J.R.Macdonald, ed., Impedance Spectroscopy: Emphasizing solid materials and systems. Wiley, New York (1987).

21. P.K. Poulouse, J.E. Morral, A.J. McEvily, Met. Trans., 5, 1393 (1974).

22. N. Birbilis, M.K. Cavanaugh, R.G. Buchheit, D.G. Harlow, R.P Wei, In proceedings symposium: Applications of Materials Science to Military Systems, Materials Science and Technology '05. Pittsburgh, PA. (2005). 


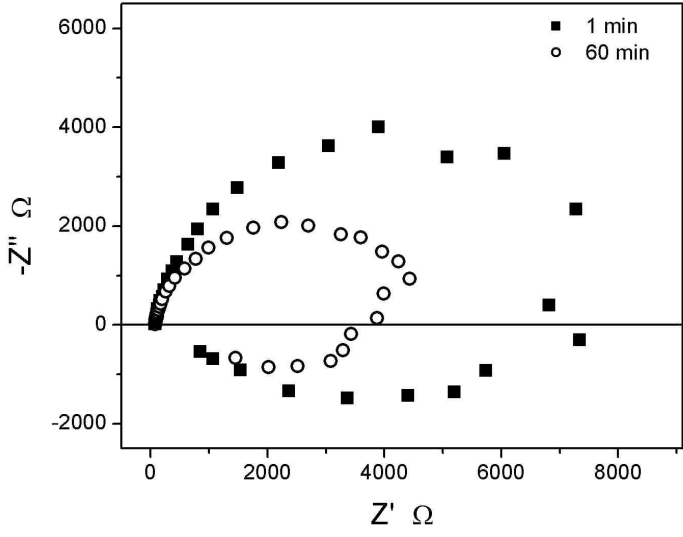

Figure 1. Nyquist plots determined for AA7075-T651 following immersion in quiescent $0.1 \mathrm{M} \mathrm{NaCl}$ for $1 \mathrm{~min}$ and 60 mins.

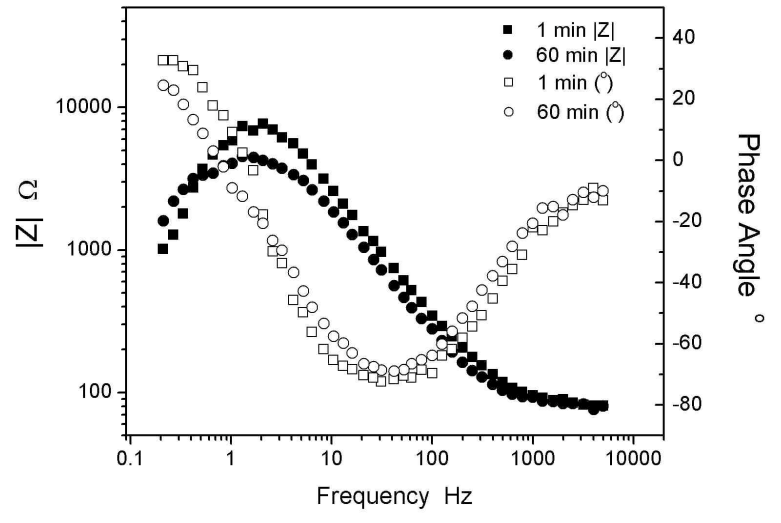

Figure 2. Bode plot corresponding to data in Fig.1.

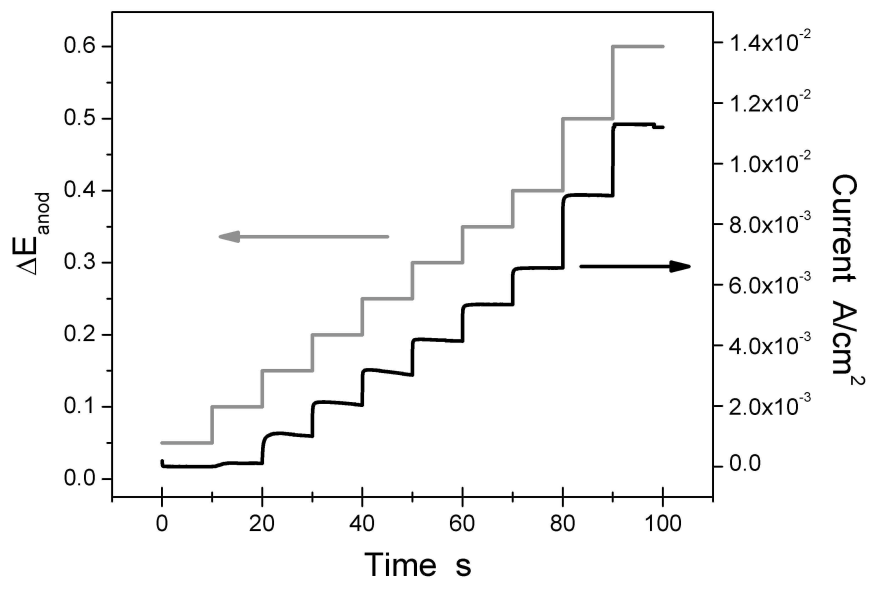

Figure 3. Current response to anodic potentio-staircase polarization in quiescent $0.1 \mathrm{M}$ $\mathrm{NaCl}$ for AA7075-T651.

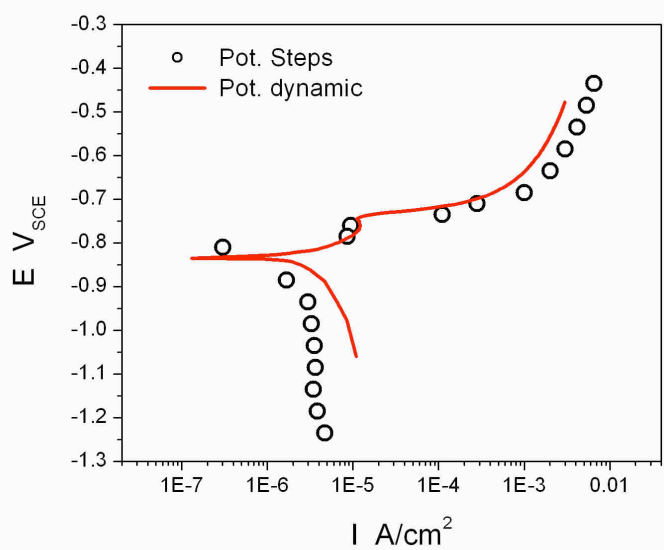

Figure 4. Potentiodynamic polarization curve and E-I relationship determined from (anodic and cathodic) potentio-staircase testing for AA7075-T651 in quiescent $0.1 \mathrm{M}$ $\mathrm{NaCl}$. 


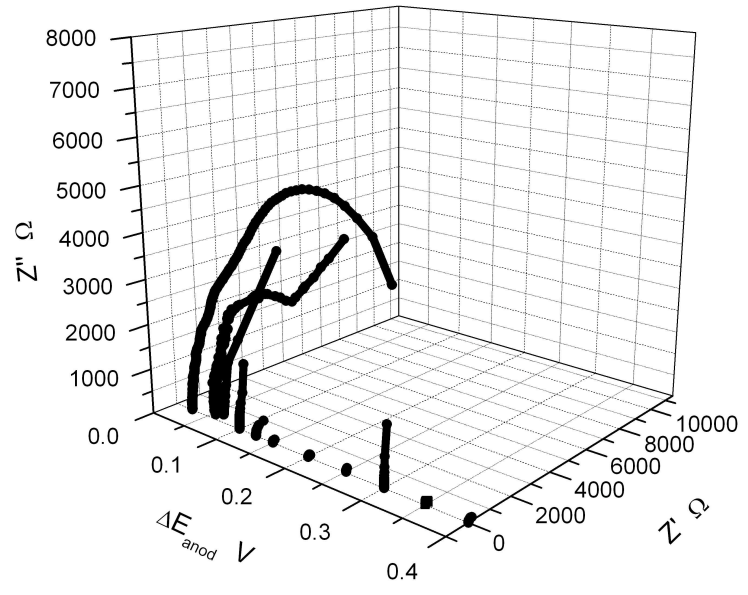

Figure 5. Potentio-impedance response of AA7075-T651 determined from Fourier analysis of data in Fig. 3. $\Delta \mathrm{E}_{\text {anod }}$ represents the amount of anodic polarization from $\mathrm{E}_{\text {corr. }}$.

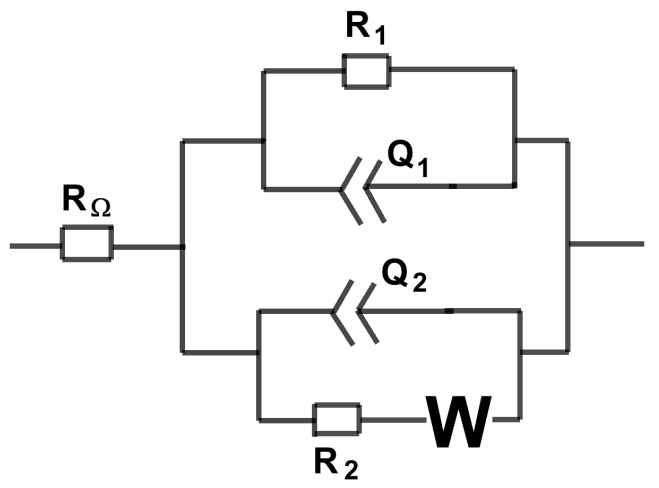

Figure 6. Equivalent circuit commonly used to define the impedance response of a metal susceptible to pitting corrosion.
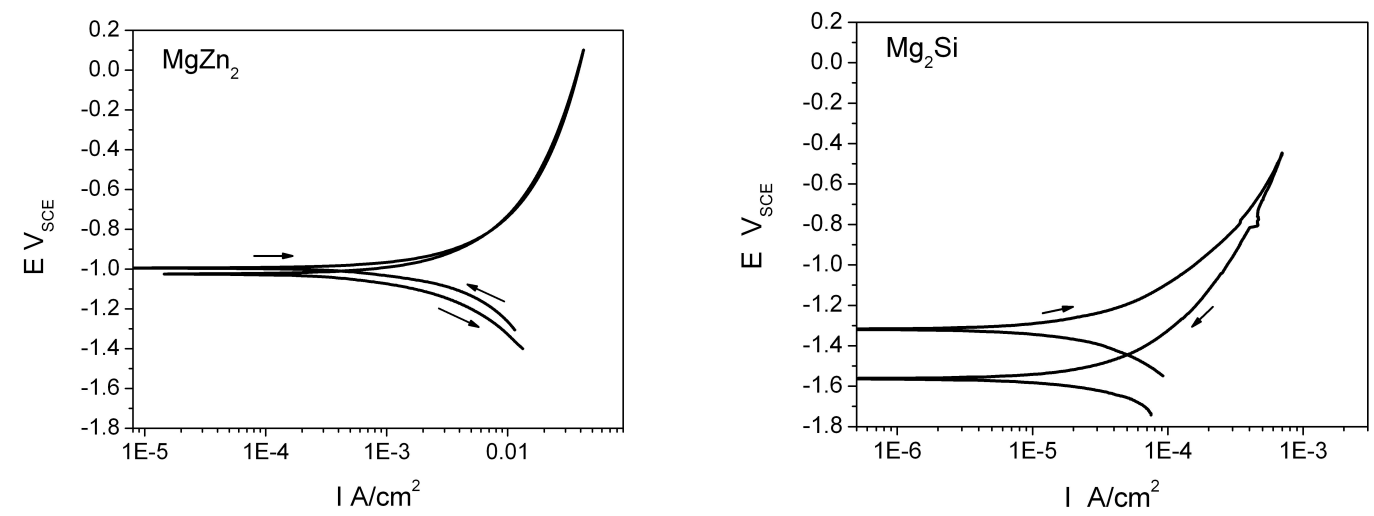

Figure 7. Potentiodynamic polarization curves for (a) $\mathrm{MgZn}_{2}$ and (b) $\mathrm{Mg}_{2} \mathrm{Si}$, in $0.1 \mathrm{M}$ $\mathrm{NaCl}$ 


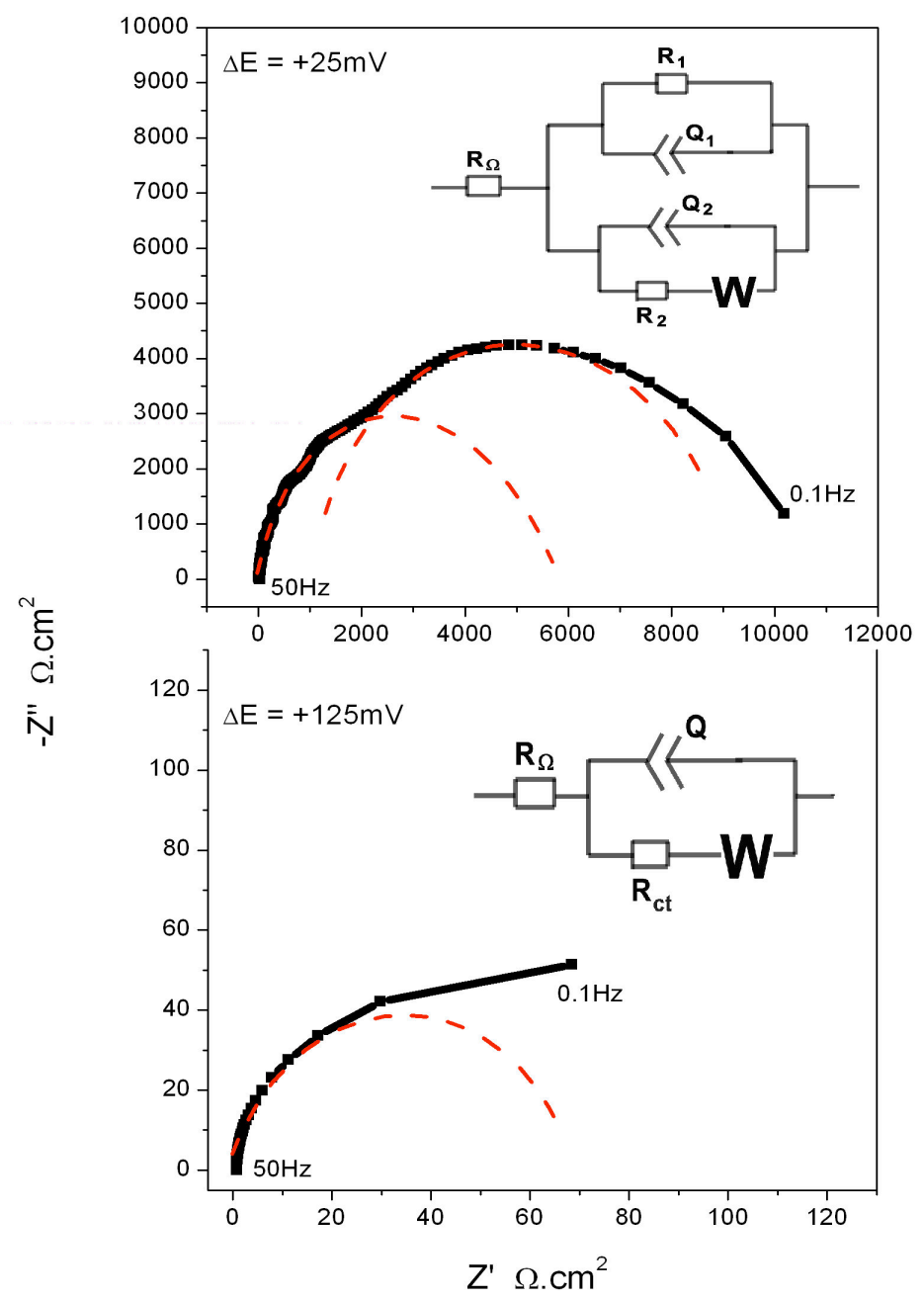

Figure 8. Nyquist representation of evaluated impedance response for AA7075-T651 at (a) $\Delta \mathrm{E}_{\text {anod }}=0.025 \mathrm{~V}$ and (b) $\Delta \mathrm{E}_{\text {anod }}=0.125 \mathrm{~V}$

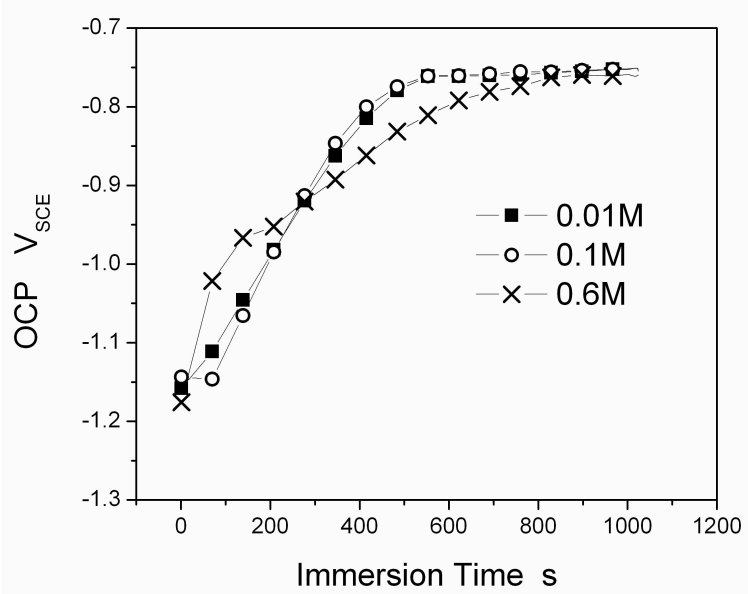

Figure 9. Open circuit potential data collected over 1000s following immersion of AA7075-T651 in quiescent $0.01,0.1$ and $0.6 \mathrm{M} \mathrm{NaCl}$ solution. 


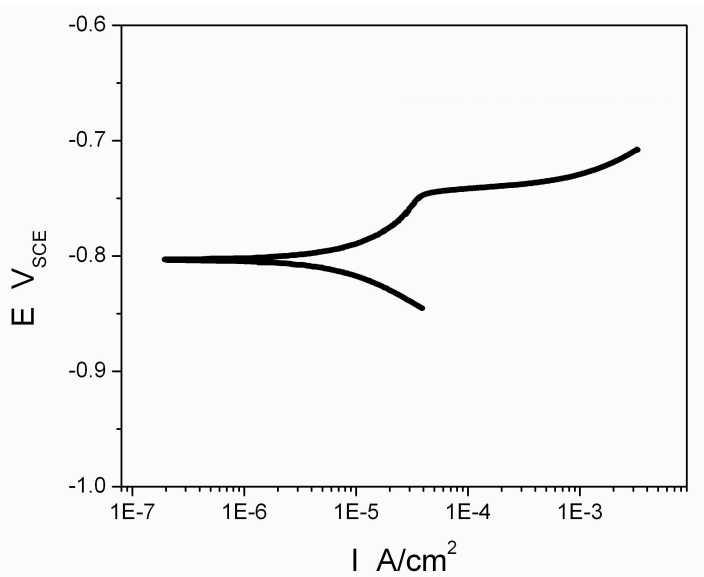

Figure 10. Potentiodynamic polarization curve for AA7075-T651 following $1000 \mathrm{~s}$ immersion in quiescent $0.1 \mathrm{M} \mathrm{NaCl}$. Breakdown is still evident.

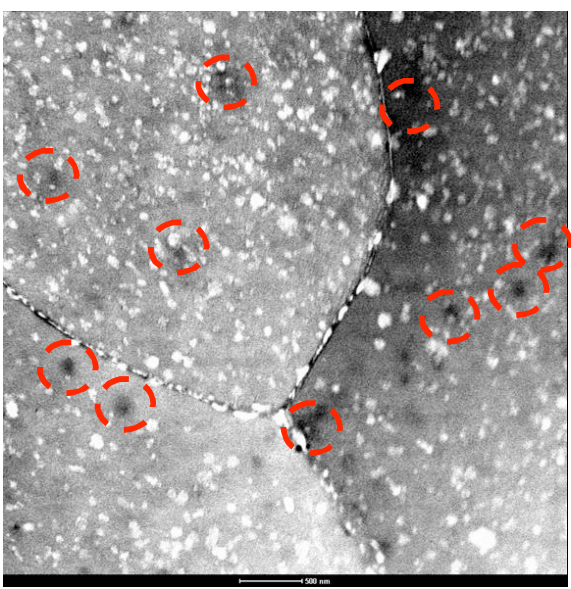

Figure 11. TEM image of AA7075-T651 following 1000s immersion in $0.1 \mathrm{M} \mathrm{NaCl}$. A selection of corroded regions emphasized by overlaid rings.

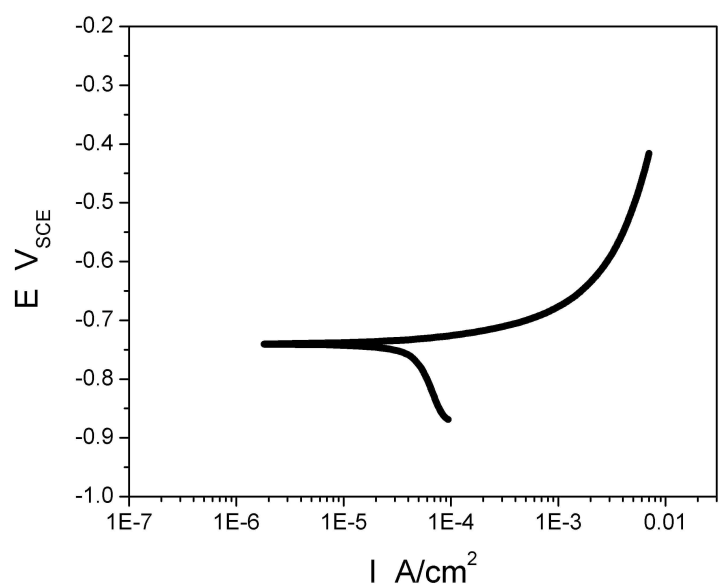

Figure 12. Potentiodynamic polarization curve for AA7075-T651 following $3600 \mathrm{~s}$ (60 mins) immersion in quiescent $0.1 \mathrm{M} \mathrm{NaCl}$. No breakdown evident. 